\title{
Identification and bioinformatics analysis of overlapping differentially expressed genes in depression, papillary thyroid cancer and uterine fibroids
}

\author{
HANXIAO TANG ${ }^{1}$ and YONGSHENG ZHANG ${ }^{2}$ \\ ${ }^{1}$ Department of Pharmacy, Affiliated Tongde Hospital of Zhejiang Chinese Medical University, Hangzhou, Zhejiang 310012; \\ ${ }^{2}$ The Diagnostic Institute of Chinese Medicine, School of Basic Medicine, Zhejiang Chinese Medical University, \\ Hangzhou, Zhejiang 310053, P.R. China
}

Received May 9, 2017; Accepted October 26, 2017

DOI: $10.3892 /$ etm.2018.6023

\begin{abstract}
It is hypothesized that there may be common characteristics between the genetic regulatory networks of different diseases. To identify these potential similarities, analysis of overlapping differentially expressed genes (DEGs) in several diseases, which are believed to be associated in traditional Chinese medicine (TCM) was performed in the present study. The gene expression profiles associated with depression, papillary thyroid carcinoma (PTC) and uterine fibroids (UF) were preliminarily analyzed using Gene Expression Omnibus 2R tools. Gene Ontology enrichment analysis, Kyoto Encyclopedia of Genes and Genomes pathway analysis and protein-protein interaction network analysis of the overlapping DEGs in depression, PTC and UF was performed. The results indicated that multiple genes, including activating transcription factor 3 and WSC domain containing 2 and the phosphoinositide 3 kinase/protein kinase $b$ signaling pathway and its downstream effectors may be common factors associated with depression, PTC and/or UF. The neuroendocrine functions of the hypothalamic-pituitary-ovarian axis and hypothalamic-pituitary-thyroid axis were also identified as being mutually associated with depression, PTC and/or UF. However, due to the limitations of DNA microassays, it is recommended that future studies take epigenetics into consideration. Further transcriptomic, methylomic and metabolomic analyses of depression, PTC and UF are also required to identify and elucidate the key associated biomarkers. In conclusion, the results of the current study shed light on the
\end{abstract}

Correspondence to: Dr Yongsheng Zhang, The Diagnostic Institute of Chinese Medicine, School of Basic Medicine, Zhejiang Chinese Medical University, 548 Binwen Road, Binjiang, Hangzhou, Zhejiang 310053, P.R. China

E-mail: alex.yszhang@zcmu.edu.cn

Key words: traditional Chinese medicine, differentially expressed genes, depression, papillary thyroid carcinoma, uterine fibroids, bioinformatics potential genetic interconnections between depression, PTC and UF, which may be beneficial for understanding their underlying coregulatory mechanisms and contributing to the development of homeotherapy based on bioinformatics prediction.

\section{Introduction}

Molecular biology methods, including DNA microarrays, are being increasingly used for high-throughput gene expression analysis and to assess the impact of genetic variation on phenotypes. As well as for the diagnosis and prognostic prediction of a single disease, DNA microarray analysis may be used to determine similarities among multiple diseases via comparative analysis, particularly when combined with Gene Ontology (GO) enrichment, Kyoto Encyclopedia of Genes and Genomes (KEGG) pathway and protein-protein interaction (PPI) analyses $(1,2)$.

Recently, the incidence rates of thyroid cancer (TC) in women have increased in several countries, including the United States and Australia (3). Although this may be associated with over-diagnosis, it is leading to an increase in the number of studies focusing on TC (4). Uterine fibroids (UF) are common benign neoplasms that occur in the uterus (5). Thyroid dysfunction may develop via the hypothalamic-pituitary-ovarian (HPO) axis due to heavy menstrual bleeding in females diagnosed with UF (6). Traditional Chinese Medicine (TCM) suggests that there is an association between TC and UF, and that these conditions are also associated with depression. However, to the best of our knowledge, there has been no systematic attempt to identify potential associations between differentially expressed genes (DEGs) associated with depression, TC and UF. Therefore, the present study analyzed the potential overlapping DEGs between these conditions using DNA microarray data, in order to biologically interpret their potential genetic associations.

In the present study, original datasets from the Gene Expression Omnibus (GEO) database (www.ncbi.nlm.nih.gov/geo/), including gene expression data series GSE12654 (depression), GSE3678 [papillary TC (PTC)] and GSE593 (UF), were evaluated. Each dataset was analyzed using GEO2R (www.nci.nlm.nih.gov/geo/geo2r/) (7), which is a GEO online tool for analyzing microarray data. The 
data of patients with depression, PTC or UF were initially compared with corresponding control groups to identify DEGs. Subsequently, the overlapping DEGs between two or three of the pathologies were screened and then subjected to GO, KEGG, PPI network analyses using various databases. By combining bioinformatics analysis methods, the present study aimed to gain insight into the potential underlying genetic interconnections between depression, PTC and UF.

\section{Materials and methods}

Microarray data. Gene expression profiles obtained from DNA microarrays were downloaded from the NCBI GEO database. The following datasets were obtained: GSE12654 (depression, $\mathrm{n}=11$; control, $\mathrm{n}=15$ ), GSE3678 (7 PTC samples, 7 control samples) and GSE593 (5 UF samples, 5 control samples). The relevant datasets were retrieved under the disease Medical Subject Headings for Homo sapiens.

Identification of DEGs. Following analysis of the different gene expression profiles in GEO2R (https://www.ncbi.nlm.nih.gov/geo/geo2r/), analyzed data were downloaded in SOFT format, converted into XLS files and screened using Microsoft Office Excel 2017 (Microsoft Corporation, Redmond, WA, USA). Genes with a $\log _{2}$ (fold-change) $\geq 1.0$ and a P-value $<0.05$ were identified as DEGs. DEGs for depression, PTC and UF were listed and the overlapping DEGs for the following combinations were subsequently screened: Depression and PTC; depression and UF; PTC and UF; and depression, PTC and UF (Fig. 1A).

Volcano plot and heat map analyses. Data normalization in microarray dataset analysis is essential to remove systematic variations (8). As a common statistical transformation, a Z-score was calculated in Microsoft Office Excel 2017 for each individual data point in a population using the following formula: Raw intensity-mean intensity/standard deviation (9). In the present study, the raw microarray data for PTC was transformed to obtain corrected expression intensities as aforementioned. Subsequently, each probe (gene) in PTC was represented by an individual dot in a volcano plot and a heat map of highly significantly expressed genes was drawn using HemI 1.0 software (http://hemi.biocuckoo.org/; Fig. 1B and C).

GO and KEGG analyses of overlapping DEGs. To identify enriched functionally associated gene groups, GO analysis was performed via the Database for Annotation, Visualization and Integrated Discovery, version 6.8 (david.ncifcrf.gov; Fig. 2) (10). As a widely applicable method, GO-based analysis aids in interpreting the biological functions and cellular components of genes and gene products (11). In addition, KEGG (www.genome.jp) provides a comprehensive database for assembling large-scale data on biosystems and for interpreting and defining molecular-level functions (12). Therefore, KEGG pathway analysis was selected via the Enrichr database (amp.pharm.mssm.edu/Enrichr/) to perform functional annotation of the overlapping DEGs of PTC and UF (Table I) (13). As few DEGs were identified between depression and the other two conditions (Fig. 1A), these data were unsuitable for GO, KEGG and PPI analyses.
PPI network analysis. With large-scale data, the STRING database 10.0 (string.embl.de/cgi/) is typically used to consolidate known and predicted PPIs (14). In the present study, to screen the significantly interactive proteins, the DEGs with a minimum required interaction score $\geq 0.4$ in STRING were selected. Molecular Complex Detection (MCODE) is an automated algorithm for identifying highly interconnected clusters in large PPI networks (15). The PPI networks were mapped via the MCODE App in Cytoscape software 3.4.0 (http://www. cytoscape.org/) (16).

\section{Results}

Identification of DEGs. Fibrinogen-like protein 2 (FGL2); activating transcription factor 3 (ATF3); ADAM metallopeptidase domain 12 (ADAM12); WSC domain containing 2 (WSCD2); and mucin 1 (MUC1) were identified as overlapping DEGs associated with depression, PTC and UF (Fig. 1A). In addition to these five genes, dual specificity tyrosine-phosphorylation-regulated kinase 2 (DYRK2); RAB9B, member RAS oncogene family pseudogene 1; thyrotropin-releasing hormone receptor (TRHR); integrin $\alpha-6$; and retinoschisin 1 were listed as the overlapping DEGs between depression and UF (Fig. 1A). Phosphodiesterase 5A (PDE5A); MYC induced nuclear antigen (MINA); angiotensin II receptor type 1 (AGTR1); and solute carrier family 14 member 1 (SLC14A1) were overlapping DEGs between depression and PTC (Fig. 1A). Notably, 157 DEGs for PTC and UF were identified. Of these, 152 directly overlapped between PTC and UF and 5 overlapped with PTC, UF and depression. This was a markedly higher number than the total overlapping DEGs between the depression and UF/PTC subsets (Fig. 1A). The volcano plot and heat map of DEGs associated with PTC are presented respectively in Fig. 1B and C. However, the heat maps of UF from the GSE593 dataset and depression from the GSE12654 dataset were not assessed as they have been previously studied $(17,18)$.

GO and KEGG analyses. The DAVID database 6.8 was used to perform GO pathway analysis of the overlapping DEGs of PTC and UF. The GO term enrichment results all met the inclusion criteria of false discovery rate $<0.01$ and $\mathrm{P}<0.01$. The top $3 \mathrm{GO}$ results with the highest enrichment scores in each category (automatically generated by DAVID) were selected and presented in Fig. 2. In addition, the top 15 results of the KEGG pathway analysis with the criteria $\mathrm{P}<0.01$ are presented in Table I, which also indicated the top enriched pathways associated with the overlapping DEGs of PTC and UF.

PPI network analysis. To screen the high confidence PPIs, a minimum required interaction score $\geq 0.4$ in the STRING database was used as a basic criterion. The high confidence PPI network was then mapped in Cytoscape (Fig. 3). This PPI network indicated that JUN proto-oncogene (JUN); AP-1 transcription factor subunit; FOS proto-oncogene; AP-1 transcription factor subunit (FOS); BCL2, apoptosis regulator (BCL2); early growth response 1 (EGR1); bone morphogenetic protein 2 (BMP2); collagen type I alpha 1 chain; fibronectin 1; signal transducer and activator of transcription 1 (STAT1); 
A
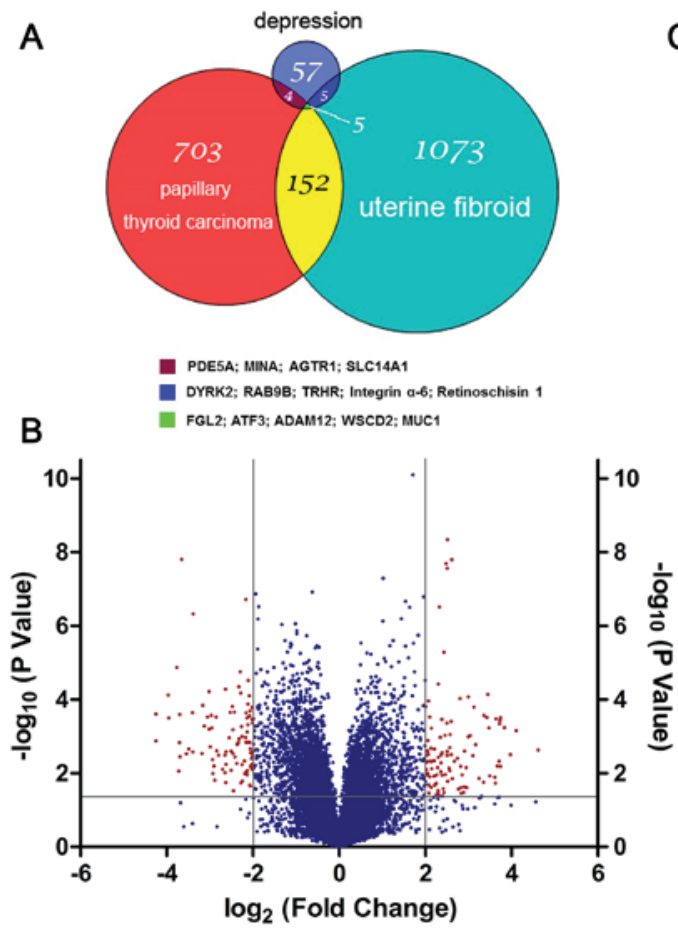

C

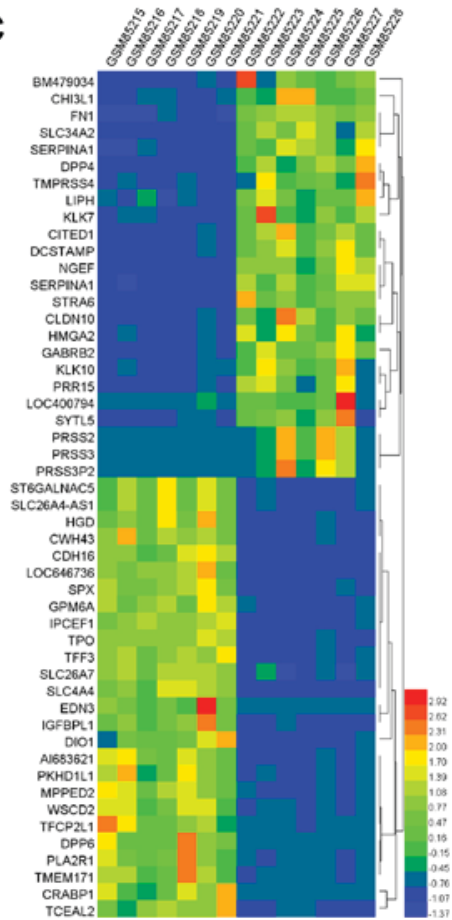

Figure 1. Venn diagram, Volcano plot and heat map. (A) Venn diagram based on the overlapping DEGs between depression, PTC and UF compared with controls. (B) Volcano plot representing the DEGs of PTC (GSE3678), satisfying the criteria of $\log _{2}$ (fold-change) value $>2$ or $<-2$ and P $<0.05$. Significantly expressed genes are represented as red dots. (C) Heat map of the 25 most upregulated and downregulated genes in PTC represented by the red dots in (B). Higher values represent upregulation. GSM85215-85221, control group; GSM85222-85228, PTC group. PTC, papillary thyroid carcinoma; UF, uterine fibroids; DEGs, differentially expressed genes.

KIT proto-oncogene receptor tyrosine kinase (KIT); and cadherin 2 were the top 10 significant hub nodes.

\section{Discussion}

PTC is the predominant histological subtype of TC and accounts for $>80 \%$ of all thyroid malignancies $(19,20)$. In TCM it is believed that PTC is associated with 'liver qi stagnation Zheng' $(21,22)$, which is thought to be primarily induced by psychological conditions, including depression and anxiety (23). In TCM, UF are thought to be induced by 'blood stasis' and are closely associated with 'qi stagnation' (24). However, it remains unknown whether there are common genetic factors between depression, PTC and UF. In the present study, the GSEs of microarray data obtained from patients with depression, PTC and UF were evaluated using GO, KEGG pathway and PPI network analyses.

DEGs of depression, PTC and UF were screened using the GEO2R tool in the GEO database. The results demonstrated a markedly higher number of overlapping DEGs between PTC and UF (157 genes) compared with that between PTC and depression (9 genes) and UF and depression (10 genes). Functional annotation implied that the DEGs between PTC and UF were primarily associated with cell adhesion/migration, extracellular structure organization, cell differentiation and cell proliferation/death. KEGG analysis manifested similar results and verified the mutual association of cell adhesion/migration-related genes. Subsequently, the 157 DEGs were screened in the STRING database according to interaction scores and the majority of proteins (100 genes) were identified to have mutual connections. A PPI network was constructed to reveal the intricate associations between these proteins. The 10 hub proteins were identified to serve a role in the following pathways: i) Immune response-relevant pathways, including toll-like receptor, $\mathrm{B} / \mathrm{T}$ cell receptor and tumor necrosis factor pathways; ii) cancer-relevant pathways, including extracellular matrix (ECM)-receptor, janus kinase/signal transducers and activators of transcription (JAK-STAT) pathways; and iii) other pathways, including the phosphoinositide 3 kinase/protein kinase B (PI3K/AKT), cyclic adenosine monophosphate, advanced glycation endproducts-receptor for advanced glycation endproducts (AGE-RAGE) and mitogen-activated protein kinase 1 (MAPK) pathways. These data indicated the associations between the functions of the top 10 hub proteins.

Adenosine monophosphate-activated protein kinase signaling is upregulated in PTC (25). Additionally, the $\mathrm{PI} 3 \mathrm{~K} / \mathrm{AKT} / \mathrm{mammalian}$ target of rapamycin (mTOR), PI3K/AKT/forkhead box O (FOXO), PI3K/AKT/phosphatase and tensin homolog deleted on chromosome ten (PTEN) and MAPK pathways have been demonstrated to be associated with TC (26-28). The PI3K-AKT/mTOR pathway has also been identified as one of the most upregulated signaling pathways in UF (29). In addition, it has previously been demonstrated that sirtuin 1 (SIRT1) is significantly associated with major depressive disorder (MDD) (30) and SIRT1, 2 and 3 bind and induce FOXO protein deacetylation (31). However, whether PI3K/AKT and downstream factors, including mTOR, FOXO and PTEN, participate in interregulatory mechanisms between depression, PTC and UF by influencing 
Table I. Kyoto Encyclopedia of Genes and Genomes pathway analysis of overlapping differentially expressed genes of papillary thyroid carcinoma and uterine fibroids.

\begin{tabular}{|c|c|c|}
\hline Term & P-values & Genes \\
\hline AGE-RAGE signaling pathway (hsa04933) & $<0.0001$ & $\begin{array}{l}\text { COL1A1; EGR1; THBD; JUN; COL3A1; STAT1; FN1; } \\
\text { BCL2; COL4A5; SELE }\end{array}$ \\
\hline Malaria (hsa05144) & $<0.0001$ & HBB; SDC1; HBA2; CD36; SELE; MET \\
\hline ECM-receptor interaction (hsa04512) & $<0.0001$ & COL1A1; FN1; SPP1; COL4A5; SDC1; CD36 \\
\hline Axon guidance (hsa04360) & $<0.0001$ & SEMA3D; SLIT1; PAK3; MET; EPHB1; EPHA3; GNAI1 \\
\hline Focal adhesion (hsa04510) & 0.0002 & COL1A1; JUN; FN1; SPP1; BCL2; COL4A5; PAK3; MET \\
\hline $\begin{array}{l}\text { Cytokine-cytokine receptor interaction } \\
\text { (hsa04060) }\end{array}$ & 0.0002 & $\begin{array}{l}\text { CCL14; BMP2; EDA; KIT; TNFRSF11B; } \\
\text { LIFR; IL1RAP; INHBA; MET }\end{array}$ \\
\hline Pathways in cancer (hsa05200) & 0.0003 & $\begin{array}{l}\text { JUN; BMP2; STAT1; KIT; FN1; BCL2; COL4A5; FOS; } \\
\text { MET; GNAI1; FGFR2 }\end{array}$ \\
\hline $\begin{array}{l}\text { Complement and coagulation cascades } \\
\text { (hsa04610) }\end{array}$ & 0.0004 & CFD; THBD; PROS1; CFI; TFPI \\
\hline Protein digestion and absorption (hsa04974) & 0.0007 & COL1A1; COL3A1; COL13A1; COL4A5; PRSS2 \\
\hline Morphine addiction (hsa05032) & 0.0007 & GABRB3; GABBR2; PDE10A; PDE8B; GNAI1 \\
\hline Cell adhesion molecules (hsa04514) & 0.0009 & CLDN10; VCAN; CDH2; SDC1; NCAM1; SELE \\
\hline Retinol metabolism (hsa00830) & 0.0017 & ADH1B; ALDH1A1; AOX1; HSD17B6 \\
\hline African trypanosomiasis (hsa05143) & 0.0025 & HBB; HBA2; SELE \\
\hline Tryptophan metabolism (hsa00380) & 0.0037 & AOX1; CYP1B1; OGDHL \\
\hline Osteoclast differentiation (hsa04380) & 0.0038 & JUN; STAT1; FOSB; TNFRSF11B; FOS \\
\hline
\end{tabular}

AGE-RAGE, advanced glycation end products-receptor for advanced glycation endproducts; ECM, extracellular matrix.

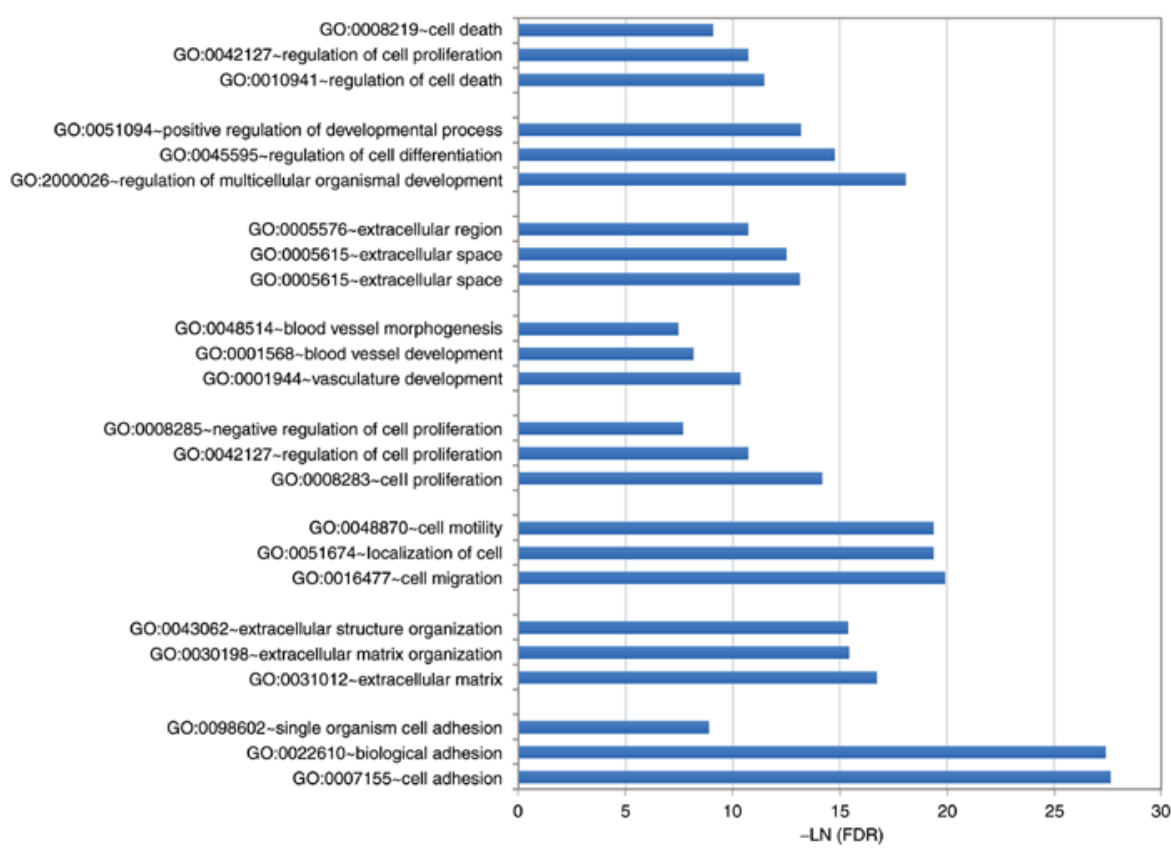

Figure 2. Gene ontology analysis of the overlapping differentially expressed genes between papillary thyroid carcinoma and uterine fibroids. -LN $(\mathrm{FDR}) ;=\log _{\mathrm{e} \text { (napier constant) }} \mathrm{FDR}$.

transcription remains unknown. Furthermore, clarification on the role served by AGE-RAGE signaling in PTC and $\mathrm{UF}$ is required, although it has recently been suggested that it is associated with the augmentation of collagen production (32).
In the present study, cGMP-specific phosphodiesterase type 5 (PDE5A) was identified as an overlapping DEG between depression and PTC. The differential expression of PDE5A has been previously reported in PTC (33) and cGMP signaling inhibits MDD development in cases of 


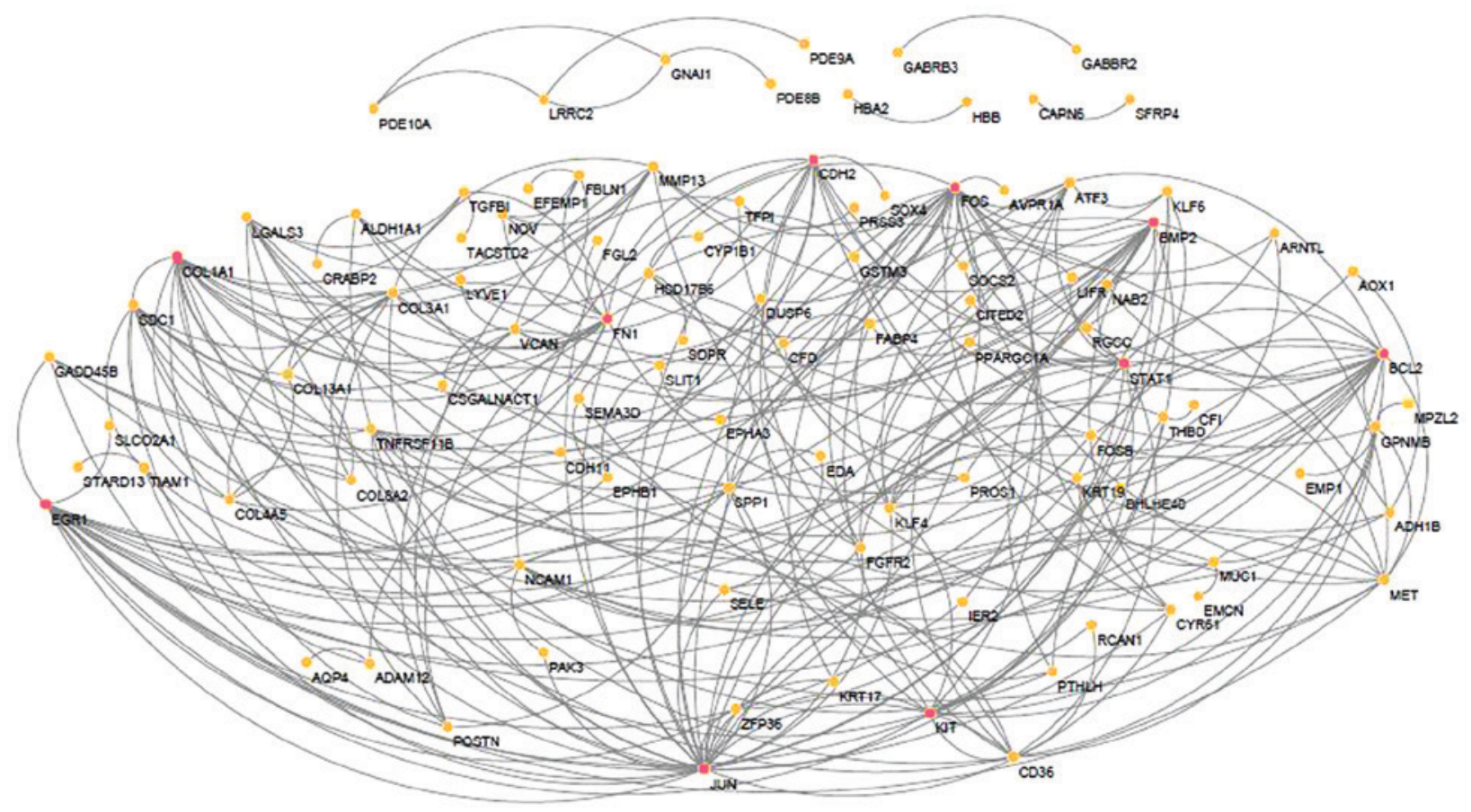

Figure 3. Constructed PPI network for the overlapping differentially expressed genes between papillary thyroid carcinoma and uterine fibroids with PPI scores $\geq 0.4$ in the Search Tool for the Retrieval of Interacting Genes/Proteins database. The top 10 hubs are represented as pink dots. PPI, protein-protein interaction.

neuroplasticity deficit (34). Genetic variation in AGTR1, which was also identified as a common DEG between depression and PTC, has been associated with depression and differences in frontotemporal morphology (35) and is a molecular marker for distinguishing between malignant and benign TC (36).

Of the overlapping DEGs associated with depression, PTC and UF in the present study, ATF3 directly interacts with several key cancer-associated proteins, including p53, E6 and androgen receptor (37) and similarly, MUC1 is aberrantly overexpressed in human breast cancer and other types of cancer $(38,39)$. ATF3 is an endoplasmic reticulum stress transcription factor, which has also been demonstrated to be significantly associated with depression- and addiction-like behavior in rats (40). In addition, as one of the 25 most downregulated genes in PTC, WSCD2 was identified to be significantly associated with extraversion of personality traits in a meta-analysis of genome-wide association studies (41). The overlapping genes identified between depression and PTC/UF are a minority and may predominantly depend on the small number of DEGs for depression. However, analysis of these DEGs may help to elucidate the underlying influence of depression on PTC/UF.

In the PPI network analysis, among the most significant hub proteins identified in the network were JUN and FOS, which induce the transcription of proteins and are associated with cell activation, proliferation, differentiation and death (42). Further functions of AP-1 include inflammatory and apoptotic activities $(43,44)$. The significant hub proteins BCL2 and STAT1 have been identified as closely associated with cancer $(45,46)$ and the PPI network also revealed interactions with JUN, FOS, KIT, EGR1 and BMP2. The results of the present study indicated that the top 10 hub proteins may serve a role in the AGE-RAGE, PI3K-Akt, ECM-receptor,
TNF and JAK-STAT signaling pathways, which corresponds with the results of the GO analysis (with regards to cell proliferation, migration, adhesion and death) and indicates potential associations between PTC and UF.

Regarding systemic disease, the impact of endocrine disorder (ED) on metabolic and physiological functions should not be understated, particularly when considering the characteristic 'domino effect' of ED. Hormones and their receptors and enzymes and transporter proteins serve a role in the intricate reactions of the endocrine system, including the hypothalamus-pituitary-thyroid (HPT) axis. In particular, hypothalamic thyrotropin-releasing hormone stimulates pituitary thyrotropin release and $80 \%$ of the triiodothyronine (T3) in the cerebral cortex is derived from the local conversion of thyroxine by deiodination (47). In the present study, TRHR was identified as an overlapping DEG in depression and UF. Active T3 exerts its function through nuclear thyroid hormone receptors (THR) and notably, deiodinase, THRA and THRB are expressed in the endometrium (48). In addition, there is a clear association between depression and thyroid function with regard to regulation by the HPT axis (49). Furthermore, interconnections between the hypothalamic-pituitary-ovarian (HPO) and HPT axes have been reported; notably hypothyroidism is closely associated with hyperprolactinemia, as well as menstrual problems, including oligomenorrhea, amenorrhoea and polymenorrhea (50). Therefore, an imbalance of hormones acting at the HPT and HPO axes may have adverse effects on mental, thyroid and/or uterine health (51).

In the present study, although only DNA microassay data analysis was used, it appeared that depression, PTC and UF may be co-associated with multiple signaling mechanisms and ED. However, due to the epigenetic effects of environmental exposure, differences in gene levels do not adequately explain the development of disease. Numerous factors, 
including genetic diathesis and environmental influences have been reported to influence the vulnerability of individuals with depression (52). UF has multiple symptoms, including abnormal uterine bleeding, infertility and urinary incontinence (53). Researchers have inferred that there is a lack of evidence to prove that total thyroidectomy and radioactive iodine therapy improves the survival rate of patients (54). Additionally, hysterectomy precludes future fertility and alternative treatments may also affect fertility (55). At least $50 \%$ of UF cases (56) and almost all microcarcinomas of PTC are considered clinically asymptomatic (57). Similarly, subclinical depression is highly prevalent and the therapeutic effects of drug and psychological treatment remain unclear (58). However, in cases of long-term depression, UF or PTC, apart from direct hormone supplement/regulation, it is unknown whether other alternative therapies aimed at regulating the neuroendocrine system or signaling networks are able to replace resective surgery such as thyroidectomy, myomectomy/hysterectomy. Recently, the identification of informative predictive biomarkers, which may be used to guide therapeutic programs for various types of cancer, has increased $(59,60)$. In the future, it is likely that insight from population-based empirical study of TCM combined with bioinformatics analysis will elucidate the potential of alternative therapies for depression, UF and PTC.

\section{Acknowledgements}

Not applicable.

\section{Funding}

The present study was supported by the National Natural Science Foundation of China (grant no. 81573700) and the Zhejiang Provincial Natural Science Foundation of China (grant no. LY16H280004).

\section{Availability of data and materials}

The analyzed data sets generated during the present study are available from the corresponding author on reasonable request.

\section{Authors' contributions}

HT collected and analyzed most of the data and drafted the initial manuscript. YZ made substantial contributions to the conception and design of the study and reviewed the manuscript.

\section{Ethics approval and consent to participate}

Not applicable.

\section{Consent for publication}

Not applicable.

\section{Competing interests}

The authors declare that they have no competing interests.

\section{References}

1. Min W, Liu C, Yang Y, Sun X, Zhang B, Xu L and Sun X: Alterations in hypothalamic-pituitary-adrenal/thyroid (HPA/HPT) axes correlated with the clinical manifestations of depression. Prog Neuropsychopharmacol Biol Psychiatry 39: 206-211, 2012

2. Sun Y, Yuan K, Zhang P, Ma R, Zhang QW and Tian XS: Crosstalk analysis of pathways in breast cancer using a network model based on overlapping differentially expressed genes. Exp Ther Med 10: 743-748, 2015.

3. Chen W, Zheng R, Baade PD, Zhang S, Zeng H, Bray F, Jemal A, Yu XQ and He J: Cancer statistics in China, 2015. CA Cancer J Clin 66: 115-132, 2016.

4. Morris LG, Sikora AG, Tosteson TD and Davies L: The increasing incidence of thyroid cancer: The influence of access to care. Thyroid 23: 885-891, 2013.

5. Stewart EA: Clinical practice. Uterine fibroids. N Engl J Med 372: 1646-1655, 2015.

6. Sweet MG, Schmidt-Dalton TA, Weiss PM and Madsen KP: Evaluation and management of abnormal uterine bleeding in premenopausal women. Am Fam Physician 85: 35-43, 2012.

7. Kim TH, Choi SJ, Lee YH, Song GG and Ji JD: Gene expression profile predicting the response to anti-TNF treatment in patients with rheumatoid arthritis; analysis of GEO datasets. Joint Bone Spine 81: 325-330, 2014.

8. Xiong H,Zhang D, Martyniuk CJ, Trudeau VL and Xia X: Using Generalized Procrustes Analysis (GPA) for normalization of cDNA microarray data. Bmc Bioinformatics 9: 25, 2008.

9. Zhao P, Hu W, Wang H, Yu S, Li C, Bai J, Gui S and Zhang Y: Identification of differentially expressed genes in pituitary adenomas by integrating analysis of microarray data. Int $\mathrm{J}$ Endocrinol 2015: 164087, 2015.

10. Liang B, Li C and Zhao J: Identification of key pathways and genes in colorectal cancer using bioinformatics analysis. Med Oncol 33: 111, 2016.

11. Gene Ontology Consortium: Gene ontology consortium: Going forward. Nucleic Acids Res 43: D1049-D1056, 2015.

12. Kanehisa M, Goto S, Sato Y, Furumichi M and Tanabe M: KEGG for integration and interpretation of large-scale molecular data sets. Nucleic Acids Res 40: D109-D114, 2012.

13. Chen EY, Tan CM, Kou Y, Duan Q, Wang Z, Meirelles GV, Clark NR and Ma'ayan A: Enrichr: Interactive and collaborative HTML5 gene list enrichment analysis tool. BMC Bioinformatics 14: 128, 2013.

14. Szklarczyk D, Franceschini A, Wyder S, Forslund K, Heller D, Huerta-Cepas J, Simonovic M, Roth A, Santos A, Tsafou KP, et al: STRING v10: Protein-protein interaction networks, integrated over the tree of life. Nucleic Acids Res 43: D447-D452, 2015.

15. Heinzel A, Mühlberger I, Fechete R, Mayer B and Perco P: Functional molecular units for guiding biomarker panel design. Biomeds Mol Biol 1159: 109-133, 2014.

16. Ding X, Wang W, Peng X and Wang J: Mining protein complexes from PPI networks using the minimum vertex cut. Tsing Sci Technol 17: 674-681, 2012.

17. Hoffman PJ, Milliken DB, Gregg LC, Davis RR and Gregg JP: Molecular characterization of uterine fibroids and its implication for underlying mechanisms of pathogenesis. Fertil Steril 82: 639-649, 2004.

18. Iwamoto K, Kakiuchi C, Bundo M, Ikeda $\mathrm{K}$ and Kato T: Molecular characterization of bipolar disorder by comparing gene expression profiles of postmortem brains of major mental disorders. Mol Psychiatry 9: 406-416, 2004.

19. Ito $Y$, Nikiforov YE, Schlumberger M and Vigneri R: Increasing incidence of thyroid cancer: Controversies explored. Nat Rev Endocrinol 9: 178-184, 2013.

20. Zhang XL and Qian LX: Ultrasonic features of papillary thyroid microcarcinoma and non-microcarcinoma. Exp Ther Med 8: 1335-1339, 2014.

21. Kehuan S, Zhengzhi W, Meiqun C, Xiaoli Z, Yinghong L, Anming W, Yu J and Zhibin R: A preliminary non-invasive salivary proteomics diagnostic model for TCM syndrome differentiation in thyroid cancer based on SELDI and bioinformatics techniques. Int Sympos Med Edu 1: 508-511, 2011.

22. Cheng F, Wang X, Song W, Lu Y, Li X, Zhang H and Wang Q: Biologic basis of TCM syndromes and the standardization of syndrome classification. J Trad Chinese Med Sci 1: 92-97, 2014. 
23. Hsu PY, Yang SH, Tsang NM, Fan KH, Hsieh CH, Lin JR, Hong JH, Lin YC, Chen HY, Yang CT, et al: Efficacy of traditional chinese medicine in xerostomia and quality of life during radiotherapy for head and neck cancer: A prospective pilot study. Evid Based Complement Altern Med: 8359251, 2016.

24. Zhu J, Xia T and Liu ZJ: Experimental study of effect of liver Qi Stagnation-psychological stress on sex hormone and immune factors in uterine leiomyomas animal model. J Liaon Univers Trad Chinese Med 9: 25, 2011 (In Chinese).

25. Vidal AP, Andrade BM, Vaisman F, Cazarin J, Pinto LF, Breitenbach MM, Corbo R, Caroli-Bottino A, Soares F, Vaisman M and Carvalho DP: AMP-activated protein kinase signaling is upregulated in papillary thyroid cancer. Eur J Endocrinol 169: 521-528, 2013.

26. Xing M: Molecular pathogenesis and mechanisms of thyroid cancer. Nat Rev Cancer 13: 184-199, 2013.

27. Faustino A, Couto JP, Pópulo H, Rocha AS, Pardal F, Cameselle-Teijeiro JM, Lopes JM, Sobrinho-Simões M and Soares P: mTOR pathway overactivation in BRAF mutated papillary thyroid carcinoma. J Clin Endocrinol Metab 97: E1139-E1149, 2012

28. Nozhat $Z$ and Hedayati M: PI3K/AKT pathway and its mediators in thyroid carcinomas. Mol Diagn Ther 20: 13-26, 2016.

29. Mcwilliams MM and Chennathukuzhi VM: Recent advances in uterine fibroid etiology. Semin Reprod Med 35: 181-189, 2017.

30. CONVERGE consortium: Sparse whole genome sequencing identifies two loci for major depressive disorder. Nature 523: 588-591, 2015

31. Tzivion G, Dobson $\mathrm{M}$ and Ramakrishnan G: FoxO transcription factors; Regulation by AKT and 14-3-3 proteins. Biochim Biophys Acta 1813: 1938-1945, 2011.

32. Peng Y, Kim JM, Park HS, Yang A, Islam C, Lakatta EG and Lin L: AGE-RAGE signal generates a specific NF- $\kappa \mathrm{B}$ RelA 'barcode' that directs collagen I expression. Sci Rep 6: 18822, 2016.

33. Kim HS, Kim DH, Kim JY, Jeoung NH, Lee IK, Bong JG and Jung ED: Microarray analysis of papillary thyroid cancers in korean. Korean J Int Med 25: 399-407, 2010.

34. Reierson GW, Guo S, Mastronardi C, Licinio J and Wong ML: cGMP signaling, phosphodiesterases and major depressive disorder. Curr Neuropharmacol 9: 715-727, 2011

35. Taylor WD, Benjamin S, McQuoid DR, Payne ME, Krishnan RR, MacFall JR and Ashley-Koch A: AGTR1 gene variation: Association with depression and frontotemporal morphology. Psychiatry Res 202: 104-109, 2012.

36. Vriens MR, Schreinemakers JM, Suh I, Guerrero MA and Clark OH: Diagnostic markers and prognostic factors in thyroid cancer. Future Oncol 5: 1283-1293, 2009.

37. Cui H, Guo M, Xu D, Ding ZC, Zhou G, Ding HF, Zhang J, Tang Y and Yan C: The stress-responsive gene ATF3 regulates the histone acetyltransferase Tip60. Nat Commun 6: 6752, 2015.

38. Kufe DW: MUC1-C oncoprotein as a target in breast cancer: Activation of signaling pathways and therapeutic approaches. Oncogene 32: 1073-1081,2013.

39. Hwang I, Kang YN, Kim JY, DO YR, Song HS and Park KU: Prognostic significance of membrane-associated mucins 1 and 4 in gastric adenocarcinoma. Exp Ther Med 4: 311-316, 2012.

40. Pavlovsky AA, Boehning D, Li D, Zhang Y, Fan X and Green TA: Psychological stress, cocaine and natural reward each induce endoplasmic reticulum stress genes in rat brain. Neuroscience 246: 160-169, 2013.

41. Lo MT, Hinds DA, Tung JY, Franz C, Fan CC, Wang Y, Smeland OB, Schork A, Holland D, Kauppi K, et al: Genome-wide analyses for personality traits identify six genomic loci and show correlations with psychiatric disorders. Nat Genet 49: 152-156, 2017.

42. Mehan S, Meena H, Sharma D and Sankhla R: JNK: A stress-activated protein kinase therapeutic strategies and involvement in Alzheimer's and various neurodegenerative abnormalities. J Mol Neurosci 43: 376-390, 2011.
43. Qiao Y, He H, Jonsson P, Sinha I, Zhao C and Dahlman-Wright K: AP-1 is a key regulator of proinflammatory cytokine $\mathrm{TNF} \alpha$-mediated triple-negative breast cancer progression. J Biol Chem 291: 18309, 2016.

44. Uluçkan Ö, Guineaviniegra J, Jimenez $M$ and Wagner EF: Signalling in inflammatory skin disease by AP-1 (Fos/Jun). Clin Exp Rheumatol 33 (4 Suppl 92): S44-S49, 2015

45. Delbridge AR, Grabow S, Strasser A and Vaux DL: Thirty years of BCL-2: Translating cell death discoveries into novel cancer therapies. Nat Rev Cancer 16: 99-109, 2016.

46. Schreiber RD, Old LJ and Smyth MJ: Cancer immunoediting: Integrating immunity's roles in cancer suppression and promotion. Science 331: 1565-1570, 2011.

47. Schroeder AC and Privalsky ML: Thyroid hormones, $\mathrm{t} 3$ and $\mathrm{t} 4$, in the brain. Front endocrinol (Lausanne) 5: 40, 2014.

48. Vissenberg R, Manders V, Mastenbroek S, Fliers E, Afink GB Ris-Stalpers C, Goddijn M and Bisschop PH: Pathophysiological aspects of thyroid hormone disorders/thyroid peroxidase autoantibodies and reproduction. Hum Reprod Update 21: 378-387, 2015.

49. Hage MP and Azar ST: The link between thyroid function and depression. J Thyroid Res 2012: 590648, 2011.

50. Tasneem A, Fatima I, Ali A, Mehmood N and Amin MK: The incidence of hyperprolactinaemia and associated hypothyroidism: Local experience from Lahore. Pak J Nuclear Med 1: 49-55, 2011.

51. Su Q, Cheng Y, Jin K, Cheng J, Lin Y, Lin Z, Wang L and Shao B: Estrogen therapy increases BDNF expression and improves post-stroke depression in ovariectomy-treated rats. Exp Ther Med 12: 1843-1848, 2016.

52. Heim C and Binder EB: Current research trends in early life stress and depression: Review of human studies on sensitive periods, gene-environment interactions and epigenetics. Exp Neurol 233: 102-111, 2012.

53. Khan AT, Shehmar M and Gupta JK: Uterine fibroids: Current perspectives. Int J Womens Health 6: 95-114, 2014.

54. Wang TS, Goffredo P, Sosa JA and Roman SA: Papillary thyroid microcarcinoma: An over-treated malignancy? World J Surg 38: 2297-2303, 2014.

55. Talaulikar VS and Manyonda IT: Ulipristal acetate: A novel option for the medical management of symptomatic uterine fibroids. Adv Ther 29: 655-663, 2012.

56. Divakar H: Asymptomatic uterine fibroids. Best Pract Res Clin Obst Gynaecol 22: 643-654, 2008.

57. Ito Y, Nikiforov YE, Schlumberger M and Vigneri R: Increasing incidence of thyroid cancer: Controversies explored. Nat Rev Endocrinol 9: 178-184, 2013.

58. Philippi CL, Motzkin JC, Pujara MS and Koenigs M: Subclinical depression severity is associated with distinct patterns of functional connectivity for subregions of anterior cingulate cortex. J Psychiatr Res 71: 103-111, 2015.

59. Gonzalez de Castro D, Clarke PA, Al-Lazikani B and Workman P: Personalized cancer medicine: Molecular diagnostics, predictive biomarkers and drug resistance. Clin Pharmacol Ther 93: 252-259, 2013

60. Winter JM, Yeo CJ and Brody JR: Diagnostic, prognostic and predictive biomarkers in pancreatic cancer. J Surg Oncol 107: $15-22,2013$.

This work is licensed under a Creative Commons Attribution-NonCommercial-NoDerivatives 4.0 International (CC BY-NC-ND 4.0) License. 